\title{
Three-particle system in a finite volume: formalism, quantization condition, spectrum and energy shift
}

\author{
Jin-Yi Pang ${ }^{1,2, *}$ \\ ${ }^{1}$ College of Science, University of Shanghai for Science and Technology, Jungong Rd. 334, 200093 Shanghai, China \\ ${ }^{2}$ Helmholtz-Institut für Strahlen- und Kernphysik and Bethe Center for Theoretical Physics, Universität Bonn, Nußallee 14-16, 53115 \\ Bonn, Germany
}

\begin{abstract}
Lattice QCD calculations provide an ab initio access to hadronic process. These calculations are usually performed in a small cubic volume with periodic boundary conditions. The infinite volume extrapolations for three-body systems are indispensable to understand many systems of high current interest. We derive the three-body quantization condition in a finite volume using an effective field theory in the particle-dimer picture. Our work shows a powerful and transparent method to read off three-body physical observables from lattice simulations. In this paper, we review the formalism, quantization condition, spectrum analysis and energy shifts calculation both for 3-body bound states and scattering states.
\end{abstract}

\section{Introduction}

A rapid progress is developed aiming to analyze of the lattice data in the three-particle sector [1-29] (for the recent review, see Ref. [30]). This is also the request of lattice simulation which has touched the three-body kinematics region. Among these approaches, our works $[17,18,20,29]$ has showed its transparent and powerful to the problem via the non-relativistic effective theory and the particle-dimer picture in a finite volume (a very similar approach was proposed in Refs. [14, 21]). It is demonstrated that effective field theory is very important to connect lattice simulation results with physical experiment data [18]. Three-body force, the critical point of the problem is fit to the lattice spectrum [17]. Efimov states, as the typical bound states are reproduced and interpreted in a finite volume within our formalism [20]. We also show that three-body threshold amplitude can be extracted from energy shifts of scattering states [29]. The equivalence to other approaches in the literature has been shown [18] as well.

The paper is arranged as followed. We show the formalism describing 3-body system in Sec. 2. The formalism is put into a finite volume and quantization condition is derived and projected in Sec. 3. We apply the quantization condition to analyze the 3-body bound states and scattering states in Sec. 4 and 5. Sec. 6 gives the conclusion and outlook.

\section{Three-body formalism}

\subsection{Three-body effective theory}

We have the effective Lagrangian including kinematics terms, 2-body operators and 3-body operators.

$$
\mathcal{L}=\mathcal{L}_{0}+\mathcal{L}_{I}^{(2-\text { body })}+\mathcal{L}_{I}^{(3-\text { body })}
$$

Non-relativistic kinematics for scalar particles is considered,

$$
\mathcal{L}_{0}=\psi^{\dagger}\left(i \partial_{0}+\frac{\nabla^{2}}{2 m}\right) \psi
$$

and the interaction operators are arranged in order of pertinent power counting,

$$
\mathcal{L}_{I}^{(2-\text { body })}=C_{0} \psi^{\dagger} \psi^{\dagger} \psi \psi+C_{2}\left(\psi^{\dagger} \stackrel{\leftrightarrow}{\nabla}^{2} \psi^{\dagger} \psi \psi+\text { h.c. }\right)+\cdots
$$

and

$$
\begin{aligned}
\mathcal{L}_{I}^{(3 \text {-body })}= & D_{0} \psi^{\dagger} \psi^{\dagger} \psi^{\dagger} \psi \psi \psi+D_{2}\left(\psi^{\dagger} \psi^{\dagger} \stackrel{\leftrightarrow}{\nabla} \psi^{\dagger} \psi \psi \psi+\text { h.c. }\right) \\
& +\cdots .
\end{aligned}
$$

Here we use $\stackrel{\leftrightarrow}{\nabla}=(\nabla-\stackrel{\leftarrow}{\nabla}) / 2$ to produce relative momentum of two particles which preserves Galilean transformation invariance.
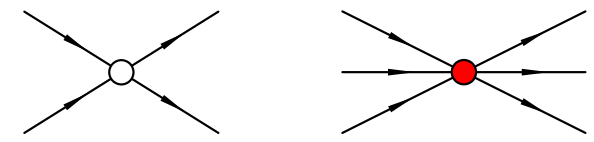

Figure 1: 2-body operator and 3-body operator

*e-mail: pang@hiskp.uni-bonn.de 


\subsection{Particle-dimer formalism}

We introduce particle-dimer formalism to make the 3-body effective theory more compact and clear. Dimer field is an auxiliary degree of freedom which describes a 2-body subsystem. For simplicity, only the scalar dimer is consid$\operatorname{ered}^{1}$. The kinematics of dimer field $T$ is added by,

$$
\mathcal{L}_{0}=\psi^{\dagger}\left(i \partial_{0}+\frac{\nabla^{2}}{2 m}\right) \psi+\sigma T^{\dagger} T .
$$

Dimer has no internal structure at tree level. The dynamics of dimer comes from its interaction with particles.

$$
\mathcal{L}_{I}^{(2-\text { body })}=T^{\dagger} \psi[f(-i \stackrel{\leftrightarrow}{\nabla})] \psi+\text { h.c. }
$$

where

$$
\psi[f(-i \stackrel{\leftrightarrow}{\nabla})] \psi=f_{0} \psi \psi+f_{2} \psi(-i \stackrel{\leftrightarrow}{\nabla})^{2} \psi+\cdots,
$$

Also the 3-body force is expressed by the form of,

$$
\mathcal{L}_{I}^{(3-\text { body })}=h_{0} T^{\dagger} T \psi^{\dagger} \psi+h_{2} T^{\dagger} T\left(\psi^{\dagger} \nabla^{2} \psi+\nabla^{2} \psi^{\dagger} \psi\right)+\cdots .
$$
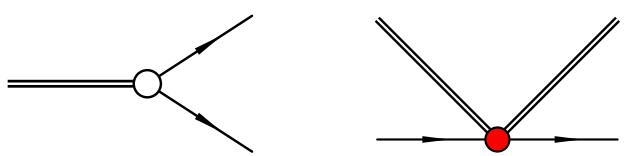

Figure 2: 2-body operator and 3-body operator in particledimer formalism

The particle-dimer formalism is totally equivalent to 3-body effective theory without any approximation. It can be seen in the path integral representation. Since dimer field is auxiliary, the complete 3-body effective theory can be reproduced by integrating dimer field out from particledimer formalism [18].

\subsection{Two-body sector}

The parameters in 2-body sector, i.e., $f_{0}, f_{2}, \cdots$ in eq.(7) should be in principal matched to 2-body physical observables, such as 2-body scattering phase shift. However, since we are focused in 3-body dynamics, these 2-body parameters can be taken account as input, either from experiment data or 2-body lattice simulation. We have demonstrated that the 2-body low-energy constants (LECs) can be encoded in phase shift (or its analytic continuation) for both particle-dimer scattering equation and 3-body quantization condition [18].

Explicitly speaking, we can write down the dimer propagator $^{2}$ in terms of,

$$
\tau(\mathbf{k} ; E)=\frac{1}{k^{*} \cot \delta\left(k^{*}\right)+\sqrt{\left(k^{*}\right)^{2}}}
$$

\footnotetext{
${ }^{1}$ The dimer with arbitrary spin can be checked in our paper [18].

${ }^{2}$ Strictly speaking, since we are using the spectator momentum $\mathbf{k}$, it is actually spectator-dimer propagator.
}

where the momentum $k^{*}$ is on-shell momentum ${ }^{3}$,

$$
\left(k^{*}\right)^{2}=\frac{3}{4} \mathbf{k}^{2}-m E-i \epsilon .
$$

At the same time, the hopping potential (see Fig. 3) is expressed by

$$
z(\mathbf{p}, \mathbf{q} ; E)=\frac{1}{p^{2}+q^{2}+\mathbf{p q}-m E} .
$$

All the 2-body parameters have been hidden. The price is that we have to input 2-body physical information, i.e., phase shift.
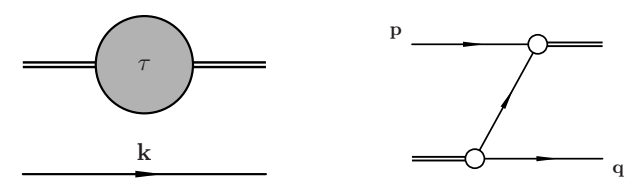

Figure 3: The left plane: dimer-propagator. The right plane: hopping potential.

\subsection{Three-body force}

The only one kind of parameters left is three-body force, i.e., $h_{0}, h_{2}, \cdots$ in eq.(8). They appear in particle-dimer scattering equation or quantization condition. Our main aim is to determine the 3-body force from lattice simulation. The total particle-dimer potential reads,

$$
Z(\mathbf{p}, \mathbf{q} ; E)=\frac{1}{p^{2}+q^{2}+\mathbf{p q}-m E}+\frac{H(\Lambda)}{\Lambda^{2}} .
$$

Here we use dimensionless parameter $H$ instead of $h_{0}, h_{2}, \cdots$ and $\Lambda$ is three-momentum cut-off ${ }^{4}$. Although the 3-body force $H$ is cut-off dependent, it does not lead to any cut-off dependence of physical result, for example, binding energy of 3-body bound state. In a finite volume, it turns out that all the energy levels are cut-off independent as well.

\subsection{Particle-dimer scattering equation}

The particle-dimer scattering equation is written down as,

$$
\begin{aligned}
& \mathcal{M}(\mathbf{p}, \mathbf{q} ; E)=Z(\mathbf{p}, \mathbf{q} ; E) \\
& \quad+8 \pi \int^{\Lambda} \frac{d^{3} k}{(2 \pi)^{3}} Z(\mathbf{p}, \mathbf{k} ; E) \tau(\mathbf{k} ; E) \mathcal{M}(\mathbf{k}, \mathbf{q} ; E) .
\end{aligned}
$$

As long as 3-body force is determined, we can solve the scattering equation. The analytic structure of particledimer scattering amplitude $\mathcal{M}$ then encodes all the 3-body dynamics.

In the infinite volume, rotational symmetry is respected. So the scattering equation can be projected to the specific angular momentum quantum number.

\footnotetext{
${ }^{3}$ For 3-body system with total momentum, $P=(E, 0)$, one particle is on-shell and has momentum $\mathbf{k}$. If another on-shell particle has momentum $|\mathbf{p}|=k^{*}$, the third particle must be on-shell as well.

${ }^{4}$ We adopt cut-off regularization to deal with ultra-violet divergence here. Other regularization schemes such as dimension regularization are available as well in the infinite volume. However, in a finite volume, cut-off scheme is most clear and natural.
} 


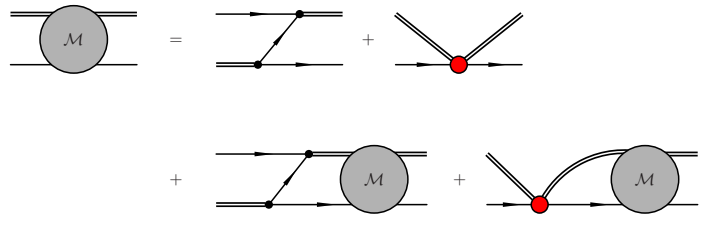

Figure 4: Particle-dimer scattering equation

\section{Projected quantization condition}

\subsection{Particle-dimer scattering equation in a finite volume}

Imposing periodical boundary condition for lattice configuration makes the momentum of the system discrete. Whereas the local LECs is non-sensitive to the volume effect. Therefore both 2-body input and 3-body force are preserved. The finite volume effects are reflected by changing loop integrals to loop sums. There are two corrections in the finite volume scattering equation, iteration part and dimer propagator.

$$
\begin{aligned}
\mathcal{M}_{L}(\mathbf{p}, \mathbf{q} ; E) & =Z(\mathbf{p}, \mathbf{q} ; E) \\
& +\frac{8 \pi}{L^{3}} \sum_{\mathbf{k}}^{\Lambda} Z(\mathbf{p}, \mathbf{k} ; E) \tau_{L}(\mathbf{k} ; E) \mathcal{M}_{L}(\mathbf{k}, \mathbf{q} ; E)
\end{aligned}
$$

where $\mathbf{k}=\frac{2 \pi}{L} \mathbf{n}, \mathbf{n} \in \mathbb{Z}^{3}$ is a quantized three-momentum in a finite volume and

$$
\tau_{L}^{-1}(\mathbf{k} ; E)=k^{*} \cot \delta\left(k^{*}\right)+S(\mathbf{k}, E)
$$

The finite volume correction for dimer propagator is

$$
S(\mathbf{k}, E)=-\frac{4 \pi}{L^{3}} \sum_{\mathbf{l}} \frac{1}{\mathbf{k}^{2}+\mathbf{l}^{2}+\mathbf{k} \mathbf{l}-m E} .
$$

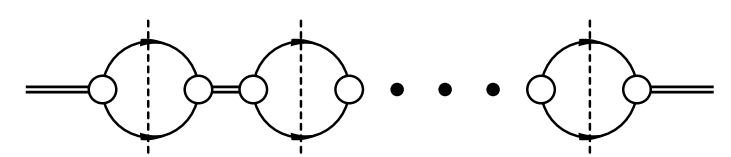

Figure 5: Finite volume correction for dimer propagator

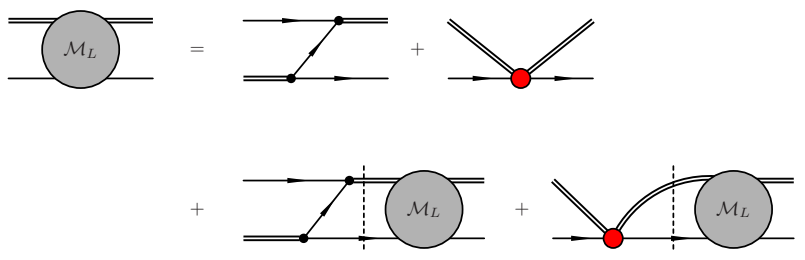

Figure 6: Finite volume scattering equation

\subsection{Quantization condition}

In a finite volume, the spectrum is discrete both below and above threshold. All the energy levels are determined by the poles of finite volume scattering amplitude $\mathcal{M}_{L}$. Based on the finite volume scattering equation, eq.(14), these poles are obtained from quantization condition,

$$
\operatorname{det}\left(\tau_{L}^{-1}-Z\right)=0
$$

Now we have written down the 3-body quantization condition. It gives the finite volume spectrum of 3-body system. Inversely, 3-body force can be fixed by plugging lattice simulation results into quantization condition. With 3-body force and pertinent 2-body input, we can solve the scattering equation in the infinite volume, i.e., eq.(13). Particle-dimer scattering amplitude $\mathcal{M}$ encodes all the 3body dynamics information.

\subsection{Octahedral group, shell structure and irreducible representation expansion}

The cubic periodical boundary condition breaks rotational symmetry in the infinite volume. The remaining symmetry for the discrete momentum configuration $\left(\mathbf{p}=\frac{2 \pi}{L} \mathbf{n}, \mathbf{n} \in\right.$ $\left.\mathbb{Z}^{3}\right)$ is octahedral group, $\mathcal{G}=O_{h}$. Combined with inversion operator, there are 48 group elements. $O_{h}$ group has 10 irreducible representations, $A_{1}^{ \pm}, A_{2}^{ \pm}, E^{ \pm}, T_{1}^{ \pm}, T_{2}^{ \pm}$.

One the other hand, with respect to the octahedral group, we define the shell structure for the discrete momenta. Shell is the set of momenta with the same magnitude. The arbitrary momentum $\mathbf{p}$ in a shell can be obtained by $O_{h}$ transformation acted on reference momentum denoted by $\mathbf{p}_{0}$, i.e., $\mathbf{p}=g \mathbf{p}_{0}, g \in \mathcal{G}$. Notice that $\mathbf{n}=(3,0,0)$ and $\mathbf{n}^{\prime}=(2,2,1)$ which have the same magnitude, although, cannot be related by any $O_{h}$ transformation. Thus they belong to two different shells. We will specify the reference momentum for each shell at the beginning. For example, shell-1 has 6 momenta, $(1,0,0),(0,1,0), \cdots$. We choose $\mathbf{p}_{0}=\frac{2 \pi}{L}(1,0,0)$. Finally, nothing depends on the choice of reference. We also define multiplicity $\vartheta(r)$ of the shell $r$, for example, $\vartheta(1)=6$.
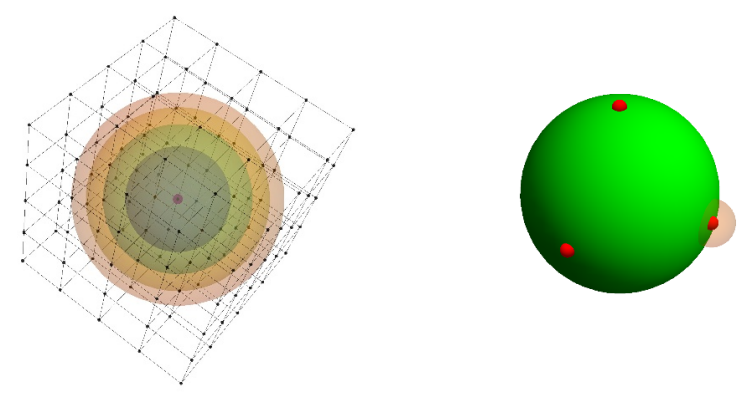

Figure 7: The left plane: Shell structure of cubic momentum configuration. The right plane: Shell-1.

Similar to the partial wave expansion corresponding to rotational symmetry, we develop the irreducible represen- 

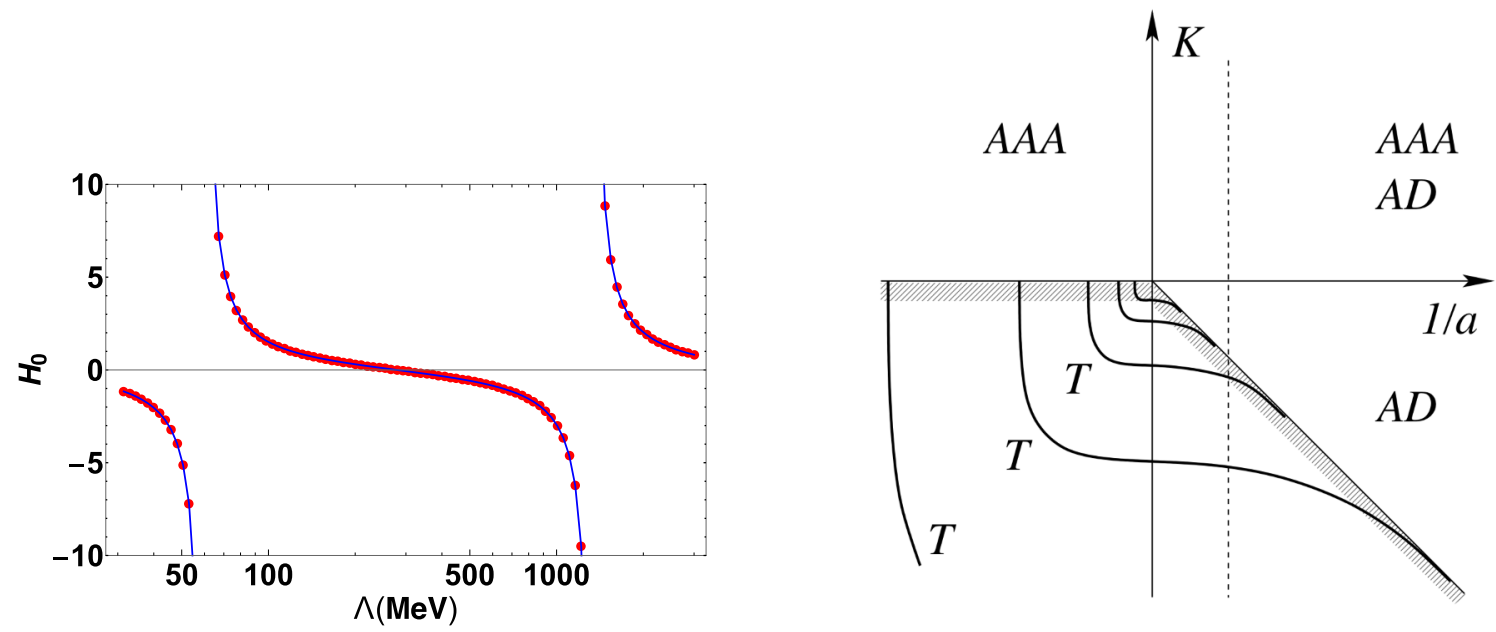

Figure 8: The left plane: 3-body force $H_{0}$ is running as momentum cut-off $\Lambda$ in the log-periodical manner. The right plane: Efimov states, $a$ is 2-body scattering length ( $1 / a=0$ corresponding to unitary limit), $K$ is 3 -body energy, $A A A$ means 3-atom state, $A D$ is the state of a spectating atom and a 2-atom bound state, $T$ means 3-body bound state, i.e., Efimov state. The vertical dashed line shows the system we are concerning.

tation expansion for the cubic symmetry ${ }^{5}$.

$$
f(\mathbf{p})=f\left(g \mathbf{p}_{0}\right)=\sum_{\Gamma} \sum_{\rho \sigma} T_{\sigma \rho}^{\Gamma}(g) f_{\rho \sigma}^{\Gamma}\left(\mathbf{p}_{0}\right)
$$

where $\Gamma=A_{1}^{ \pm}, A_{2}^{ \pm}, E^{ \pm}, T_{1}^{ \pm}, T_{2}^{ \pm}$, and $T_{\sigma \rho}^{\Gamma}(g)$ are the matrices of the irreducible representations [20]. Inversely, we have

$$
f_{\rho \sigma}^{\Gamma}\left(\mathbf{p}_{0}\right)=\frac{s_{\Gamma}}{G} \sum_{g \in \mathcal{G}}\left(T_{\sigma \rho}^{\Gamma}(g)\right)^{*} f\left(g \mathbf{p}_{0}\right)
$$

where $G=48$ is the total number of elements in the group $\mathcal{G}$ and $s_{\Gamma}$ is the dimension of the representation $\Gamma$.

For the finite volume scattering equation, the potential is projected as

$$
\begin{aligned}
Z(\mathbf{p}, \mathbf{q} ; E) & =Z\left(g \mathbf{p}_{0}(s), g^{\prime} \mathbf{q}_{0}(r) ; E\right) \\
& =\sum_{\Gamma, \rho \sigma, \lambda} \frac{s_{\Gamma}}{G} T_{\rho \sigma}^{(\Gamma)}(g) Z_{\sigma \lambda}^{(\Gamma)}(s, r ; E) T_{\rho \lambda}^{(\Gamma) *}\left(g^{\prime}\right)
\end{aligned}
$$

and

$$
Z_{\sigma \lambda}^{\Gamma}(s, r ; E)=\sum_{g \in \mathcal{G}}\left(T_{\lambda \sigma}^{\Gamma}(g)\right)^{*} Z\left(\mathbf{p}_{0}(s), g \mathbf{q}_{0}(r) ; E\right) .
$$

The dimer propagator is projected automatically,

$$
\tau_{L}(s ; E)=\tau_{L}\left(\mathbf{p}_{0}(s) ; E\right)=\tau_{L}(\mathbf{p}(s) ; E) .
$$

Finally, we arrive at projected quantization condition,

$$
\operatorname{det}\left(\tau_{L}(s)^{-1} \delta_{r s} \delta_{\sigma \rho}-\frac{8 \pi \vartheta(s)}{G L^{3}} Z_{\sigma \rho}^{\Gamma}(r, s)\right)=0 .
$$

This equation determines the energy levels in the irreducible representation $\Gamma$.

\footnotetext{
${ }^{5}$ The expansion is complete based on

$$
\sum_{g \in \mathcal{G}}\left(T_{\rho \sigma}^{\Gamma}(g)\right)^{*} T_{\rho^{\prime} \sigma^{\prime}}^{\Gamma^{\prime}}(g)=\delta_{\Gamma \Gamma^{\prime}} \delta_{\rho \rho^{\prime}} \delta_{\sigma \sigma^{\prime}} \frac{G}{s_{\Gamma}}
$$

and

$$
\sum_{\Gamma, \rho \sigma} \frac{s_{\Gamma}}{G}\left(T_{\rho \sigma}^{\Gamma}(g)\right)^{*} T_{\rho \sigma}^{\Gamma}\left(g^{\prime}\right)=\delta_{g g^{\prime}}
$$
}

\section{Three-body bound states}

\subsection{Efimov states}

We apply 3-body quantization condition on the system where Efimov states exist. The 3-body force plays an essential role for the forming of Efimov states which are the typical 3-body bound states.

The 2-body physical information as input is encoded in phase shift giving the following effective range expansion,

$$
p \cot \delta=-\frac{1}{a}+\frac{1}{2} r p^{2}+\cdots, \quad a \gg r .
$$

The pertinent 3-body force generates 3-body bound state whatever 2-body interaction is attractive or repulsive. By solving eq.(13), we produce Efimov states via a logperiodical 3-body force $H_{0}$ (see Fig. 8).

In the system with positive 2-body scattering length, i.e., $a>0$, there exists a 2-body bound state with binding momentum ${ }^{6} \sim 1 / a$. It induces an additional threshold above which $A D$ states (the state of a spectating particle and a 2-body bound state) emerge. The threshold is an effective 2-body threshold ( $A D$ threshold) contrast to 3-body threshold above which $A A A$ states (the state of three particles) emerge. We find two bound states in the 3-body sector. The shallow one is close to $A D$ threshold from below while the deeper one is far away relatively (see Fig. 8). All the physics are encoded in scattering equation in the infinite volume, i.e., eq.(13).

\subsection{Efimov states in a finite volume}

In a finite volume, we apply 3-body quantization condition (23) by inputting the 3-body force which has been fixed in the infinite volume. The two 3-body bound states in a finite

\footnotetext{
${ }^{6}$ Here we define binding momentum $\kappa=\sqrt{m\left|E_{B}\right|}, E_{B}$ is the energy of the bound state.
} 
volume are both reproduced (see Fig. 9). They are in the irreducible representation $A_{1}^{+}$corresponding to $S$-wave in the infinite volume.

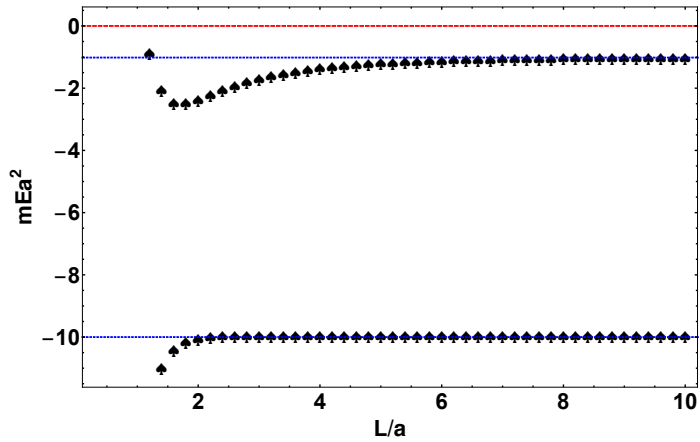

Figure 9: The two Efimov states in a finite volume

\subsection{Energy shifts of bound states}

There are two different scenarios for the 3-body bound states in the infinite volume.

- Effective 2-body limit: $\kappa^{2}-a^{-2} \ll \kappa^{2}$;

- 3-body limit: $\kappa a \gg 1$.

Here $\kappa$ is 3-body binding momentum defined by $\kappa=$ $\sqrt{m\left|E_{B}\right|}$ and $E_{B}$ is bound state pole. These two cases have different energy shifts in a finite volume, respectively.

We firstly relate the finite volume amplitude $\mathcal{M}_{L}$ in eq.(14) to the infinite volume amplitude $\mathcal{M}$ in eq.(13) by

$$
\begin{aligned}
& \mathcal{M}_{L}(\mathbf{p}, \mathbf{q} ; E)=\mathcal{M}(\mathbf{p}, \mathbf{q} ; E) \\
& \quad+8 \pi \int \frac{d^{3} k}{(2 \pi)^{3}} \mathcal{M}(\mathbf{p}, \mathbf{k} ; E) \delta \tau(\mathbf{k} ; \mathbf{E}) \mathcal{M}_{L}(\mathbf{k}, \mathbf{q} ; E)
\end{aligned}
$$

where

$$
\delta \tau(\mathbf{k} ; \mathbf{E})=\sum_{\mathbf{n} \neq 0} e^{i \mathbf{n k} L} \tau(\mathbf{k} ; E)+\left(\text { high order in } \frac{1}{L}\right) .
$$

The infinite volume amplitude $\mathcal{M}$ can be expanded around the bound state pole $E_{B}$,

$$
\mathcal{M}(\mathbf{p}, \mathbf{q} ; E)=\frac{\psi(\mathbf{p}) \psi^{\dagger}(\mathbf{q})}{E-E_{B}}+\mathrm{reg} .
$$

The residue at the pole gives the wave-function $\psi(\mathbf{p})$ of Efimov state as an effective 2-body system of dimer field and the spectator. The energy shift, therefore, can be calculated by

$$
\begin{aligned}
\Delta E & =E_{L}-E_{B} \\
& =8 \pi \sum_{\mathbf{n} \neq 0} \int \frac{d^{3} k}{(2 \pi)^{3}} \psi^{\dagger}(\mathbf{k}) \tau\left(\mathbf{k} ; E_{B}\right) e^{i \mathbf{n k} L} \psi(\mathbf{k}) .
\end{aligned}
$$

In the effective 2-body limit, eq.(28) gives that

$$
\frac{\Delta E}{\left|E_{B}\right|} \sim \frac{C_{2}}{\kappa L} e^{-\frac{2}{\sqrt{3}} \sqrt{\kappa^{2}-a^{-2}} L}
$$

which is consistent with 2-body Lüscher formula. While in the 3-body limit, we have energy shift,

$$
\frac{\Delta E}{\left|E_{B}\right|} \sim \frac{C_{3}}{(\kappa L)^{3 / 2}} e^{-\frac{2}{\sqrt{3}} \kappa L} .
$$

$C_{3}$ is proportional to asymptotic normalization coefficient $|\mathcal{A}|^{2}$. It turns out that in the unitary limit, $a \rightarrow \infty$, the ratio $C_{3} /|\mathcal{A}|^{2}=-96.351$ is universal [2].

The general 3-body bound state is the mixing of the two limits. By analyzing the 3-body contribution and effective 2-body contribution in the energy shifts of two bound states (see Fig. 10), we find that:

- The shallow bound state is dominated by effective 2body scenario.

- 3-body picture and effective 2-body picture are both important in the deeper bound state.

\section{Three-body scattering states}

\subsection{Avoided level crossing}

Since there are two thresholds in the system, $A D$ threshold and 3-body threshold, the scattering states in a finite volume $^{7}$ tend to these two thresholds in the infinite volume limit, respectively. Consequently, we can find both effective 2-body states and 3-body states above threshold in the lattice spectrum (see Fig. 11). All the states are determined by quantization condition (23) which is projected on $A_{1}^{+}$-irrep.

Avoided level crossing happens between the two kinds of scattering states. In 2-body lattice spectrum, avoided level crossing usually implies the existence of resonance. Reproducing this behavior in 3-body spectrum is a good sign for us to interpret 3-body resonance in the future simulation.

\subsection{Energy shifts of scattering states}

The scattering states distribute around free lines which are the non-interacting energy levels. The energy shifts of 3body states above threshold can be expressed in the series of $1 / L$, i.e.,

$$
\begin{aligned}
m E_{L}= & N\left(\frac{2 \pi}{L}\right)^{2}+\frac{\#}{L^{3}}+\frac{\#}{L^{4}}+\frac{\#}{L^{5}} \\
& +\frac{\#}{L^{5}} \log \left(\frac{m L}{2 \pi}\right)+\frac{\#}{L^{6}}+\cdots
\end{aligned}
$$

where $N(2 \pi / L)^{2}$ is the free energy level. The high order coefficients can be obtained by solving quantization condition (23) perturbatively.

The expansions around free lines also indicate that the symmetry representations of these spectra are related to the specific momentum shell. For example, the lowest energy level is related to shell-0, i.e., $\mathbf{p}=0$. Shell-0 contains only $A_{1}^{+}$-irrep which means the ground state must be in $A_{1}^{+}$. The 1 st excited states are related to shell-1 including

\footnotetext{
${ }^{7}$ In the infinite volume, the states above threshold are continuous. Whereas in a finite volume, they are discrete.
} 

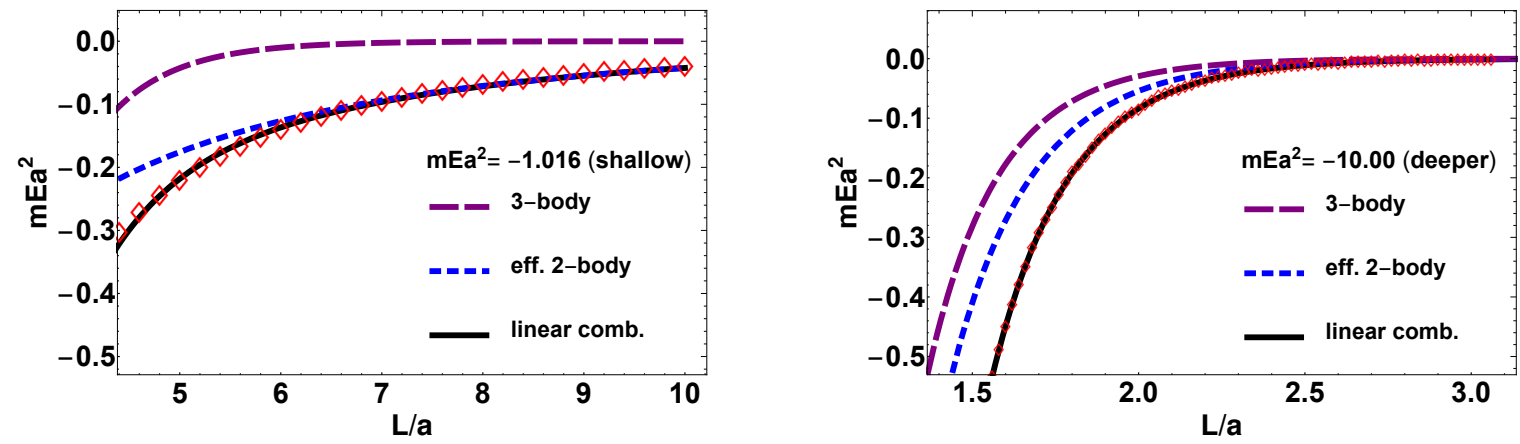

Figure 10: The left plane: energy shift of the shallow bound state. The right plane: energy shift of the deeper bound state.

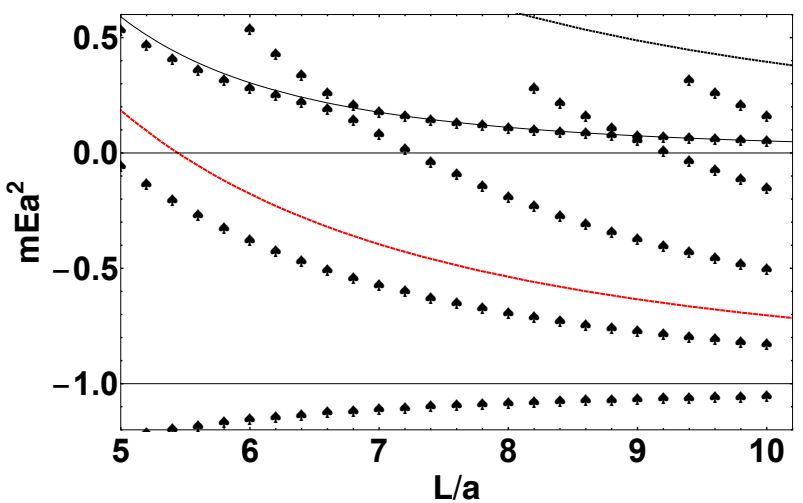

Figure 11: The 3-body lattice spectrum in $A_{1}^{+}$. $A D$ threshold is located at -1.0 while 3 -body threshold at 0 . Red dashed line indicates the spectrum for free state of a 2body bound state and a spectator. Black dashed line is the free line for the 3 free particles. Black solid line is the theoretical calculation for the 3-body ground state.

$A_{1}^{+}, E^{+}$and $T_{1}^{-}$-irrep. Therefore we are supposed to find three spectra around shell-1. But due to the identical particles principal, $T_{1}^{-}$spectrum does not exist actually.

The quantization condition

$$
\operatorname{det}\left(\tau^{-1}-Z\right)=0
$$

can be solved perturbatively around shell- $r$ in the form of

$$
\left(\tau^{-1}-Z\right)_{r r}=\sum_{k \neq r} Z_{r k} \tau_{k} Z_{k r}+\sum_{k, l \neq r} Z_{r k} \tau_{k} Z_{k l} \tau_{l} Z_{l r}+\cdots
$$

where $k, l$ are both shell numbers. They also denote the matrix indices of $\tau, Z$ and so on. Both sides of eq.(33) are expanded with respect to $1 / L$, so the energy shifts can be calculated order by order.

The energy shift of the ground state (which resides in the $A_{1}^{+}$-irrep) is:

$$
m E_{L}=\left(\frac{2 \pi}{L}\right)^{2} \frac{g_{0}}{L}\left(1+\frac{g_{1}}{L}+\frac{g_{2}}{L^{2}}+\frac{g_{3}}{L^{3}} \log \frac{m L}{2 \pi}+\frac{g_{4}}{L^{3}}+\cdots\right)
$$

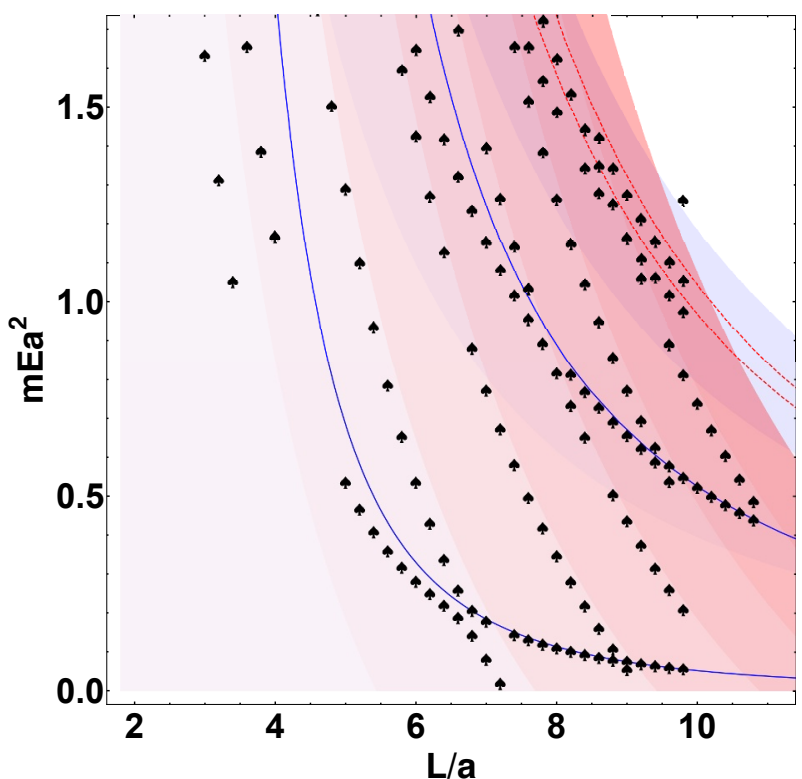

Figure 12: The lower blue line is the theoretical calculation for energy shifts of ground states up to $O\left(1 / L^{6}\right)$. The upper blue line is for the excited states in $A_{1}^{+}$up to $O\left(1 / L^{6}\right)$ as well. The two red lines are the calculation for higher excited states at the next to leading order, that is $O\left(1 / L^{3}\right)$.

where

$$
\begin{aligned}
& g_{0}=\frac{3}{\pi} a, \\
& g_{1}=2.837297480 a, \\
& g_{2}=9.725330808 a^{2}, \\
& g_{3}=8 \pi\left(\frac{2 \sqrt{3}}{\pi}-\frac{8}{3}\right) a^{3}, \\
& g_{4}=\left(-5.159159617+6 \pi\left(\frac{r}{a}\right)-8 \pi\left(\frac{\hat{\mathcal{M}}}{a^{2}}\right)\right) a^{3} .
\end{aligned}
$$

Here $a$ is 2-body scattering length and $r$ is 2-body scattering effective range. In $g_{4}$, the 3 -body threshold amplitude $\hat{\mathcal{M}}$ emerges. This 3-body physical quantity can be fit to lattice spectrum up to the order of $1 / L^{6} . \hat{\mathcal{M}}$ also appears 
in the energy shift of the 1 st excited state in the $A_{1}^{+}$-irrep,

$m E_{L}=\left(\frac{2 \pi}{L}\right)^{2}\left(1+\frac{h_{0}}{L}\left(1+\frac{h_{1}}{L}+\frac{h_{2}}{L^{2}}+\frac{h_{3}}{L^{3}} \ln \frac{m L}{2 \pi}+\frac{h_{4}}{L^{3}}+\cdots\right)\right)$

with

$h_{0}=\frac{10}{\pi} a$,

$h_{1}=0.279070 a$,

$h_{2}=\left(8.494802+\frac{7 \pi^{2}}{5}\left(\frac{r}{a}\right)\right) a^{2}$,

$h_{3}=\frac{27}{5} \times 8 \pi\left(\frac{2 \sqrt{3}}{\pi}-\frac{8}{3}\right) a^{3}$,

$h_{4}=\left(-172.001650+83.745841\left(\frac{r}{a}\right)-\frac{27}{5} \times 8 \pi\left(\frac{\hat{\mathcal{M}}}{a^{2}}\right)\right) a^{3}$.

However, $\hat{\mathcal{M}}$-dependence of the energy shift of the 1 st excited state in the $E^{+}$-irrep disappears because $E^{+}$corresponds to high derivative force while $\hat{\mathcal{M}}$ is non-derivative quantity.

$$
m E_{L}=\left(\frac{2 \pi}{L}\right)^{2}\left(1+\frac{h_{0}^{\prime}}{L}\left(1+\frac{h_{1}^{\prime}}{L}+\frac{h_{2}^{\prime}}{L^{2}}+\frac{h_{4}^{\prime}}{L^{3}}+\cdots\right)\right),
$$

with

$$
\begin{aligned}
& h_{0}^{\prime}=\frac{4}{\pi} a, \\
& h_{1}^{\prime}=2.984094 a, \\
& h_{2}^{\prime}=\left(3.001706+\frac{\pi^{2}}{2}\left(\frac{r}{a}\right)\right) a^{2}, \\
& h_{4}^{\prime}=\left(-28.89478538+54.584571\left(\frac{r}{a}\right)\right) a^{3} .
\end{aligned}
$$

\section{Conclusion}

We have reviewed the effective field theory describing 3body system. Particle-dimer formalism shows its powerful to analyze the 3-body problems both in the infinite volume and a finite volume. 3-body quantization condition is derived. Considering the cubic symmetry for the lattice configuration, we also develop the irreducible representation expansion to project quantization condition on specific quantum number. The finite volume spectrum in the corresponding irreducible representation is determined consequently. The physical pictures of 3-body bound states in the infinite volume can be interpreted via energy shifts in a finite volume. For 3-body scattering states, avoided level crossing is observed and energy shifts are calculated perturbatively by expanding the quantization condition. 3body threshold amplitude emerging in the energy shifts up to $O\left(1 / L^{6}\right)$ can be extracted in principal from lattice simulation.

Our formalism needs further development including introducing twisted boundary condition, relativistic kinematics and so on. The application on realistic system, such as triton and Roper resonance is on-going as well.
Acknowledgments: The work is done in collaboration with H.-W. Hammer and A. Rusetsky. The authors would like to thank M. Döring, M. Mai, Ulf.-G. Meißner and J.-J. $\mathrm{Wu}$ for the collaboration on the most of works. We would also like to thank S. Beane, W. Detmold, M. Hansen, F. Romero-López, M. Savage, S. Sharpe, C. Thomas and C. Urbach for interesting discussions. JYP acknowledges the support from the DFG (CRC 110 "Symmetries and the Emergence of Structure in QCD”).

\section{References}

[1] K. Polejaeva and A. Rusetsky, Eur. Phys. J. A 48 (2012) 67, arXiv:1203.1241 [hep-lat].

[2] U.-G. Meißner, G.-Rios and A. Rusetsky, Phys. Rev. Lett. 114 (2015) 091602 Erratum: Phys. Rev. Lett. 117 (2016) 069902, arXiv:1412.4969 [hep-lat].

[3] P. Guo, Phys. Rev. D 95 (2017) 054508, arXiv:1607.03184 [hep-lat].

[4] P. Guo and V. Gasparian, Phys. Lett. B 774 (2017) 441, arXiv:1701.00438 [hep-lat].

[5] R. A. Briceño and Z. Davoudi, Phys. Rev. D 87 (2013) 094507, arXiv:1212.3398 [hep-lat].

[6] M. T. Hansen and S. R. Sharpe, Phys. Rev. D 90 (2014) 116003, arXiv:1408.5933 [hep-lat].

[7] M. T. Hansen and S. R. Sharpe, Phys. Rev. D 92 (2015) 114509, arXiv:1504.04248 [hep-lat].

[8] M. T. Hansen and S. R. Sharpe, Phys. Rev. D 93 (2016) 014506, arXiv:1509.07929 [hep-lat].

[9] M. T. Hansen and S. R. Sharpe, Phys. Rev. D 93 (2016) 096006, arXiv:1602.00324 [hep-lat].

[10] M. T. Hansen and S. R. Sharpe, Phys. Rev. D 95 (2017) 034501, arXiv:1609.04317 [hep-lat].

[11] R. A. Briceño, M. T. Hansen and S. R. Sharpe, Phys. Rev. D 95 (2017) 074510, arXiv:1701.07465 [hep-lat].

[12] S. Kreuzer and H.-W. Hammer, Phys. Lett. B 694 (2011) 424, arXiv:1008.4499 [hep-lat].

[13] S. Kreuzer and H.-W. Hammer, Eur. Phys. J. A 43 (2010) 229, arXiv:0910.2191 [nucl-th].

[14] S. Kreuzer and H.-W. Hammer, Phys. Lett. B 673 (2009) 260, arXiv:0811.0159 [nucl-th].

[15] S. Kreuzer and H.-W. Grießhammer, Eur. Phys. J. A 48 (2012) 93, arXiv:1205.0277 [nucl-th].

[16] S. R. Sharpe, Phys. Rev. D 96 (2017) 054515, arXiv:1707.04279 [hep-lat].

[17] H.-W. Hammer, J.-Y. Pang and A. Rusetsky, JHEP 1709 (2017) 109, arXiv:1706.07700 [hep-lat].

[18] H.-W. Hammer, J.-Y. Pang and A. Rusetsky, JHEP 1710 (2017) 115, arXiv:1707.02176 [hep-lat].

[19] Y. Meng, C. Liu, U.-G. Meißner and A. Rusetsky, Phys. Rev. D 98 (2018) 014508, arXiv:1712.08464 [hep-lat].

[20] M. Döring, H.-W. Hammer, M. Mai, J.-Y. Pang, A. Rusetsky and J. Wu, Phys. Rev. D 97 (2018) no.11, 114508, arXiv:1802.03362 [hep-lat].

[21] M. Mai and M. Döring, Eur. Phys. J. A 53 (2017) 240, arXiv:1709.08222 [hep-lat]. 
[22] R. A. Briceño, M. T. Hansen and S. R. Sharpe, arXiv:1803.04169 [hep-lat].

[23] F. Romero-López, A. Rusetsky and C. Urbach, Eur. Phys. J. C 78 (2018) no.10, 846, arXiv:1806.02367 [hep-lat].

[24] R. A. Briceño, M. T. Hansen and S. R. Sharpe, arXiv:1810.01429 [hep-lat].

[25] M. Mai and M. Döring, arXiv:1807.04746 [hep-lat].

[26] P. Guo and T. Morris, arXiv:1808.07397 [hep-lat].

[27] P. Guo, M. Döring and A. P. Szczepaniak, Phys. Rev. D 98 (2018) 094502, arXiv:1810.01261 [hep-lat].

[28] T. D. Blanton, F. Romero-López and S. R. Sharpe, arXiv:1901.07095 [hep-lat].

[29] J.-Y. Pang, J.J. Wu, H.-W. Hammer, Ulf.-G. Meißner and A. Rusetsky Phys. Rev. D 99 (2019) no.7, 074513, arXiv:1902.01111 [hep-lat].

[30] M. T. Hansen and S. R. Sharpe, arXiv:1901.00483 [hep-lat].

[31] M. Lüscher, Commun. Math. Phys. 105 (1986) 153.

[32] K. Huang and C. N. Yang, Phys. Rev. 105 (1957) 767.
[33] T. T. Wu, Phys. Rev. 115 (1959) 1390.

[34] S. Tan, Phys. Rev. A 78 (2008) 013636, arXiv:0709.2530 [cond-mat.stat-mech].

[35] S. R. Beane, W. Detmold and M. J. Savage, Phys. Rev. D 76 (2007) 074507, arXiv:0707.1670 [hep-lat].

[36] W. Detmold and M. J. Savage, Phys. Rev. D 77 (2008) 057502 arXiv:0801.0763 [hep-lat].

[37] E. Braaten and H.-W. Hammer, Phys. Rept. 428 (2006) 259 [cond-mat/0410417].

[38] V. Bernard, M. Lage, U.-G. Meißner and A. Rusetsky, JHEP 0808 (2008) 024, arXiv:0806.4495 [hep-lat].

[39] M. Göckeler, R. Horsley, M. Lage, U.-G. Meißner, P. E. L. Rakow, A. Rusetsky, G. Schierholz and J. M. Zanotti, Phys. Rev. D 86 (2012) 094513, arXiv:1206.4141 [hep-lat].

[40] F. Romero-López, A. Rusetsky and C. Urbach, Phys. Rev. D 98 (2018) 014503, arXiv:1802.03458 [hep-lat].

[41] H. W. Hammer and T. Mehen, Phys. Lett. B 516 (2001) 353, ,nucl-th/0105072.

[42] C. Ji, D. R. Phillips and L. Platter, Annals Phys. 327 (2012) 1803, arXiv:1106.3837 [nucl-th] 